\title{
A Step-Wise Approach for Integrating QoS throughout Software Development
}

\author{
Stéphanie Gatti, Emilie Balland, and Charles Consel \\ Thales Airborne Systems / University of Bordeaux / INRIA, France \\ first-name.last-name@inria.fr
}

\begin{abstract}
When developing real-time systems such as avionics software, it is critical to ensure the performance of these systems. In general, deterministic Quality of Service (QoS) is guaranteed by the execution platform, independently of a particular application. For example, in the avionics domain, the ARINC 664 standard defines a data network that provides deterministic QoS guarantees. However, this strategy falls short of addressing how the QoS requirements of an application get transformed through all development phases and artifacts. Existing approaches provide support for QoS concerns that only cover part of the development process, preventing traceability.

In this paper, we propose a declarative approach for specifying QoS requirements that covers the complete software development process, from the requirements analysis to the deployment. This step-wise approach is dedicated to control-loop systems such as avionics software. The domainspecific trait of this approach enables the stakeholders to be guided and ensures QoS requirements traceability via a tool-based methodology.
\end{abstract}

Keywords: Quality of Service, Domain-Specific Design Language, ToolBased Development Methodology, Generative Programming.

\section{Introduction}

Non-functional requirements are used to express the quality to be expected from a system. For real-time systems such as avionics, it is critical to guarantee this quality, in particular time-related performance properties. For example, the avionics standard ARINC 653 defines a Real-Time Operating System (RTOS) providing deterministic scheduling [3] and thus ensuring execution fairness between applications. Another example is the ARINC 664 that defines Avionics Full DupleX switched Ethernet (AFDX), a network providing deterministic Quality of Service (QoS) for data communication [4]. In this domain, deterministic QoS is generally ensured at the execution platform level (e.g., operating systems, distributed systems technologies, hardware specificities), independently of a particular application. When addressing the QoS requirements of a given application, these platform-specific guarantees are not sufficient.

There exist numerous specification languages to declare QoS requirements at the architectural level [1]. Initially, these languages were mostly contemplative. Several recent approaches also provide support to manage specific aspects

D. Giannakopoulou and F. Orejas (Eds.): FASE 2011, LNCS 6603, pp. $217-231,2011$.

(C) Springer-Verlag Berlin Heidelberg 2011 
(e.g., coherence checking [10, prediction [20], monitoring [17]). These approaches are generally dedicated to a particular development stage, leading to a loss of traceability (i.e., the ability to trace all the requirements throughout the development process). In the avionics certification processes [11125], traceability is mandatory for both functional and non-functional requirements. The functional traceability is usually ensured by systematic development methodologies such as the $\mathrm{V}$-model that guides stakeholders from the requirements analysis to the system deployment. Similarly, QoS should be fully integrated into the development process as it is a crosscutting concern [19].

In this paper, we propose a step-wise QoS approach integrated through all development phases and development artifacts. This approach is dedicated to control-loop systems. Control-loop systems are systems that sense the external environment, compute data, and eventually control the environment accordingly. This kind of systems can be found in a range of domains, including avionics, robotics, and pervasive computing. For example, in the avionics domain, a flight management application is a control-loop system that (1) senses the environment for location and other navigation information, (2) computes the trajectory and (3) modifies the wings configuration accordingly. The contributions of this paper can be summarized as follows.

A step-wise QoS approach dedicated to control-loop systems. We propose a step-wise approach that systematically processes QoS requirements throughout software development. This integrated approach is dedicated to control-loop systems, allowing to rely on a particular architectural pattern and thus enhancing the design and programming support level for non-functional aspects. In this paper, we focus on time-related performance but the approach could be generalized to other non-functional properties (e.g., CPU or memory consumption).

Requirements Traceability. In the avionics domain, the traceability of both functional and non-functional requirements is critical [11. In our approach, the traceability is ensured by the systematic propagation of constraints derived from the QoS declarations and applied to each development step.

A tool-based methodology. Our approach has been integrated into DiaSuite, a tool-based development methodology dedicated to control-loop systems [8]. DiaSuite is based on a dedicated design language that we have enriched with timerelated performance properties. This non-functional extension has been used to offer verification and programming support at each development stage.

Experiments in the avionics domain. Our approach has been applied to the development of various avionics applications, including a flight management system and a collision avoidance system. These experiments have demonstrated that our step-wise approach can effectively guide the avionics certification process.

\section{Background and Working Example}

This section presents a working example used throughout this paper. This presentation is done in the context of the DiaSuite development methodology [8]. 
We choose a control-loop system from the avionics domain: a simplified version of an aircraft guidance application, controlling the trajectory of an aircraft by correcting the configurations of ailerons.

\subsection{Overview of the DiaSuite Approach}

The DiaSuite approach is a tool-based methodology dedicated to control-loop systems. DiaSuite provides support for each development stage (from design to deployment) as depicted in Figure 1.

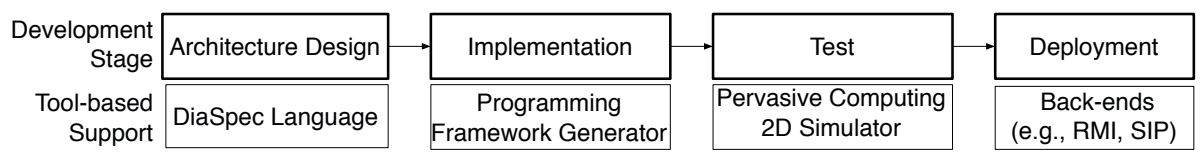

Fig. 1. The DiaSuite tool-based development process

During the design stage, the DiaSpec language allows to design an application using an architectural pattern dedicated to control-loop systems. This specific architectural pattern comprises four layers of components: (1) sensors obtain raw data from the environment; (2) contexts process data and provide highlevel information; (3) controllers use this information to control actuators; (4) actuators impact the environment. The sensors and actuators are the two facets of entities corresponding to devices, whether hardware or software, deployed in an environment.

This specification guides the developer throughout the development process. The DiaSpec compiler generates a Java programming framework dedicated to the application. This framework precisely guides the programmer during the implementation stage by providing high-level operations for entity discovery and component interactions. Based on these declarations, a simulator dedicated to pervasive computing environments is used to test and simulate the system. Then, the DiaSuite back-ends enable the deployment of an application by targeting a specific distributed systems technology such as RMI, SIP or Web Services.

\subsection{Aircraft Guidance Application}

The aircraft guidance application uses two sensors for computing the actual aircraft trajectory: the inertial reference unit, providing the localization, and the air data unit, supplying such measurements as the airspeed and the angle of attack. The synchronization of both information sources allows to compute the actual aircraft trajectory. This trajectory is then compared to the flight plan entered by the pilot and used for controlling and correcting ailerons by the automatic pilot, if necessary.

Following the DiaSuite development methodology, the first step identifies the devices involved in the aircraft guidance application using a domain-specific taxonomy of entities, as can be found in the aeronautics literature. In this example, 


\begin{tabular}{|c|c|c|c|}
\hline & \begin{tabular}{|l|} 
AutomaticPilot \\
controlAilerons \\
\end{tabular} & & Actuator Layer \\
\hline & $\begin{array}{c}\text { Guidance } \\
\text { Controller }\end{array}$ & & Controller Layer \\
\hline ActualTrajectory & Jorrec & & Context Layer \\
\hline $\begin{array}{c}\text { localization } \\
\text { InertialReferenceUnit }\end{array}$ & $\begin{array}{c}\text { airData } \\
\text { AirDataUnit }\end{array}$ & $\begin{array}{c}\text { plannedTrajectory } \\
\text { FlightPlanDataBase }\end{array}$ & Sensor Layer \\
\hline
\end{tabular}

Fig. 2. A data-flow view of the aircraft guidance application

we have identified four entities: the inertial reference unit, the air data unit, the flight plan database and the automatic pilot. The second step of the methodology consists of designing the application using DiaSpec. The system description is illustrated in Figure 2, making explicit the four component layers of DiaSpec.

In the example, the InertialRef erenceUnit sensor provides the current localization of the aircraft. The AirDataUnit sensor supplies several air data such as the airspeed and the angle of attack. All these data are sent to the ActualTrajectory context that is responsible for computing the current trajectory of the aircraft. This information is then sent to the TrajectoryCorrection context component. When receiving a new trajectory, the TrajectoryCorrection component gets the planned trajectory (from the flight plan initially entered by the pilot) from the FlightPlanDatabase component. By comparing these information sources, it computes trajectory corrections that are sent to the GuidanceController component, responsible for controlling ailerons through the AutomaticPilot actuator.

The avionics certification process requires this trajectory readjustment to be time-bounded. In the next section, we show how the DiaSuite approach, enriched with time-related properties, can guide the development of such critical applications.

\section{QoS throughout Software Development}

This section presents how QoS requirements can be systematically processed throughout software development.

\subsection{Requirements Analysis and Functional Specification}

In software development methodologies, the requirements analysis stage identifies the users' needs. Then, the functional specification stage identifies the main functionalities to be fulfilled by the application to satisfy the users' requirements. In the avionics domain, each of these functionalities is generally associated to a functional chain [26], representing a chain of computations, from sensors to actuators. 
The aircraft guidance system has a unique functional chain, whose execution should take less than 3 seconds, according to our expert at Thales Airborne Systems. In avionics, such time constraints, directly associated to a specific functional chain, is referred to as Worst Case Execution Time (WCET) 1 . If the design process involves refinement steps such as the identification of functional chain segments, the WCETs can be further refined. For example, we can identify a functional chain segment corresponding to the computation of the actual trajectory (from the sensors feeding the ActualTrajectory context). This chain segment can be reused in other applications, e.g., displaying the actual trajectory on the navigation display unit. According to our expert, this chain segment must not take more than 2 seconds to execute.

\subsection{Architecture Design}

During the architecture design stage, the functional chains are decomposed into connected components. The architect can then refine the WCET of the functional chain on time-related constraints at the component level.

In the DiaSuite architectural pattern, the data flow between two components can be realized using two interaction modes: by pulling data (one-to-one synchronous interaction mode with a return value) or by pushing data to event subscribers (asynchronous publish/subscribe interaction mode). Pull interactions are typically addressed by a response time requirement as is done in Web Services [17. Push interactions raise a need to synchronize two or more input events of a component. This need is addressed by introducing a freshness requirement on the input event values. This requirement is in the spirit of synchronization skew in the multimedia domain [18]. In addition to the freshness constraint, we define the bounded synchronization-time constraint that authorizes desynchronization during a bounded time, avoiding diverging synchronization strategies.

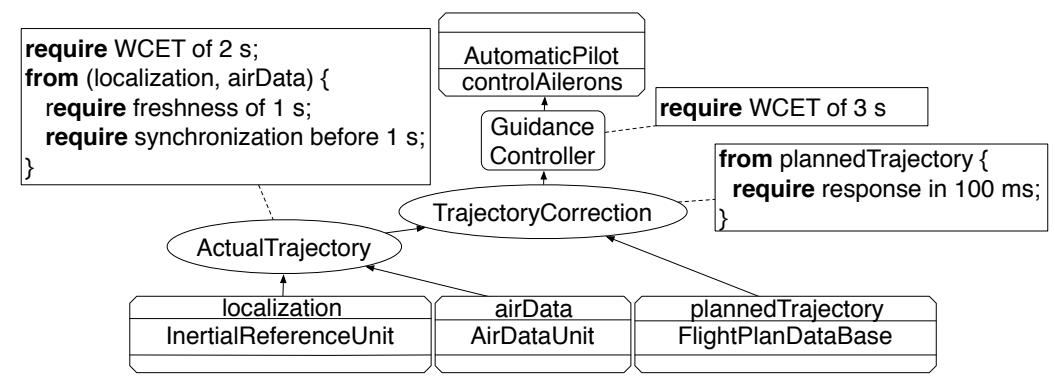

Fig. 3. Architecture of the working example, enriched with QoS contracts

Figure 3 shows the QoS contracts associated to each component in the flight guidance application. The WCETs associated to the functional chain of the

${ }^{1}$ This usage of WCET is only loosely related to the notion of WCET as documented in the literature for hard real-time systems. 
aircraft guidance and to the trajectory computing chain segment are mapped to the GuidanceController and ActualTrajectory components, respectively. The WCET of the functional chain of the aircraft guidance is refined into (1) a freshness constraint of 1 second between localization and airData with an equal bounded synchronization-time constraint (since the WCET is not compatible with a longer desynchronization time); and (2) a response time of 100 milliseconds of FlightPlanDataBase. The WCET associated to the functional chain of the aircraft guidance is translated into a QoS contract, attached to GuidanceController as controllers are generally dedicated to a given functional chain.

The QoS contracts are introduced as an extension of DiaSpec. Figure 4 shows an extract from the DiaSpec specification.

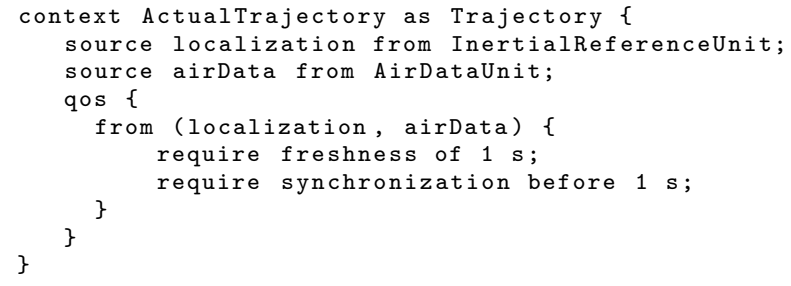

Fig. 4. DiaSpec declaration of the ActualTrajectory component

The ActualTrajectory component is declared with the context keyword, and returns values of type Trajectory. This component processes two sources of information: localization and airData. These sources are declared using the source keyword that takes a source name and a class of entities. Then, the QoS contract declared using the qos keyword defines freshness and synchronization constraints between localization and airData. This domain-specific approach guides the stakeholders when adding QoS requirements by automatically enforcing the conformance between the QoS contracts and the functional constraints.

\subsection{Implementation}

The DiaSuite approach includes a compiler that generates a dedicated programming framework from a DiaSpec description. Our approach enriches this process by generating runtime-monitoring support from QoS declarations. At the implementation level, QoS requirements on components become runtime verifications that rely on the communication methods of the generated programming framework. Monitoring mechanisms are encapsulated into component containers that ensure that the response time and the freshness requirements are respected. The approach based on containers allows a separation of concerns between functional and non-functional requirements because a container is only in charge of 
intercepting calls for monitoring requirements, and forwarding the calls to the functional component. If a QoS contract is violated, the container throws specific exceptions ResponseTimeException or SynchronizationException. The treatment of such exceptions is left to the developer. It may involve any number of actions, including logging or reconfiguration [17. DiaSuite provides declarative support at the architectural level to design exceptional treatments [21], preventing the application to be bloated and entangled with error-handling code.

The code corresponding to the response time requirement is straightforward. It is based on a timer that calculates the elapsed time between the request and the response. The more elaborate part concerns the synchronization defined by the automaton depicted in Figure 5. Suppose we want to synchronize data1 and data2 values. When receiving the first data (S2 and S4 states), the container activates the $t$ and $t$ ' timers for measuring respectively synchronization time and freshness $\left(t, t^{\prime}:=0\right)$. While synchronization time is not reached $(t<=t s)$, the container waits for fresh data. If the other data is received before the freshness time has elapsed, the container pushes both data to the functional component (S5 final state). Otherwise, if the freshness is not respected ( $\left.t^{\prime}>t f\right)$, the data is rejected. This is not considered as an error state since we authorize desynchronization for a finite period. Thereby the container waits for new values (S3 state). If the synchronization time has elapsed ( $t>t s)$, the synchronization is aborted and a SynchronizationException is thrown (error state).

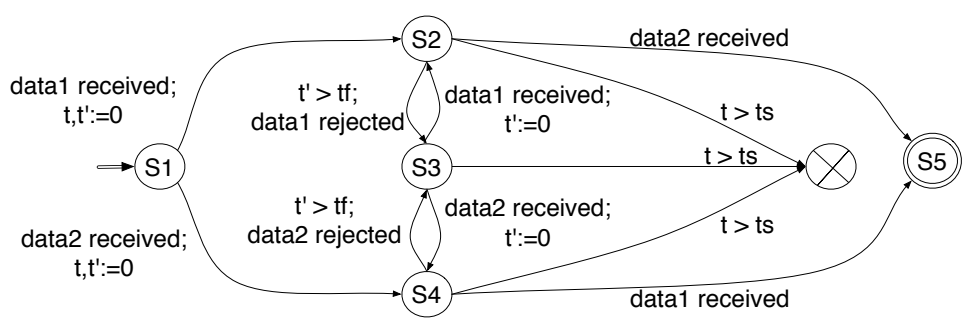

Fig. 5. Synchronization automaton

\subsection{Deployment}

Our approach offers support for predicting the performance of an application by injecting deployment parameters, such as distributed systems technologies, platform and hardware characteristics. By taking advantage of their QoS characteristics (e.g., the guaranteed deterministic timing of the AFDX network [4]), it is possible to refine the time-related requirements generated from the QoS declarations, and thus to compare several deployment configurations. In particular, it allows technologies to be selected according to their time-related properties.

This prediction tool takes numerical constraints generated from the QoS declarations as input. Then, an external constraint solver [9] checks whether a configuration respects the WCET of the functional chain and predicts constraints 
to the other architectural elements. In the next section, we detail how these numerical constraints are generated and propagated throughout the software development process.

\section{QoS Requirements Traceability}

The requirements traceability is the guarantee for each requirement to be traced back to its origin (i.e., a QoS declaration), by following its propagation in the software development process. By generating numerical constraints from the QoS declarations, our step-wise approach allows the traceability of QoS requirements during software development. This approach is summarized in Figure 6 .

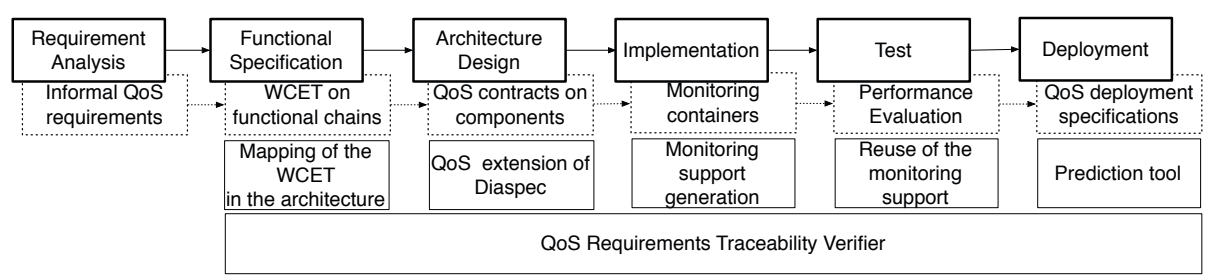

Fig. 6. Development process of Figure 1 extended with QoS concerns

At each development step, QoS declarations are translated into numerical constraints that are fed to the verifier of requirements traceability. This verifier propagates these numerical constraints between the development stages, and checks whether no new constraint invalidates constraints from preceding stages. In this section, we detail how these numerical constraints are generated and propagated.

\subsection{From Functional Specification to Architecture Design}

From functional specification to architecture design, requirements on functional chains (or chain segments) are refined into requirements on components. By generating numerical constraints, it is possible to ensure that the refinement does not invalidate requirements from the previous stage. Doing so amounts to checking whether the constraint system is still satisfied. The generation of these numerical constraints is inspired by the Defour et al.'s work [10] and relies on the DiaSuite architectural pattern. It consists of automatically translating the QoS contracts presented in Section 3 into numerical equations specifying time relationships between the components. For example, let us detail how these equations are generated for the aircraft guidance application.

WCET on functional chains and chain segments. The functional chain that controls the trajectory has a WCET of 3 seconds, represented by the contract attached to GuidanceController. This leads to the following numerical constraints: 
T_wcet_GuidanceController $<=3$;

T_wcet_GuidanceController =

T_provide_GuidanceController +

T_com(GuidanceController, AutomaticPilot);

The first equation represents the WCET associated to GuidanceController. The second equation refines this functional chain into a sequence of two functions: one for computing orders (the GuidanceController chain segment), and one for communicating these orders to the automatic pilot. Thus, the global time is the sum of the T_provide_GuidanceController time and the communication time between GuidanceController and AutomaticPilot (denoted by the T_com function). The T_provide_GuidanceController time corresponds to the chain segment between the moment when the InertialReferenceUnit or AirDataUnit sensor sends a value and the moment when GuidanceController issues orders to AutomaticPilot.

Similarly, the T_provide_GuidanceController time can also be refined into the time of GuidanceController to compute orders, the communication time between TrajectoryCorrection and GuidanceController, and the global time associated to the chain segment of TrajectoryCorrection:

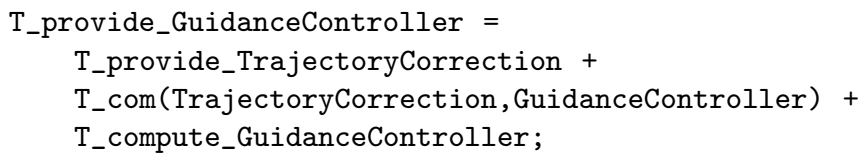

Response Time. The time associated to TrajectoryCorrection can be refined with respect to its relation with ActualTrajectory and FlightPlanDataBase:

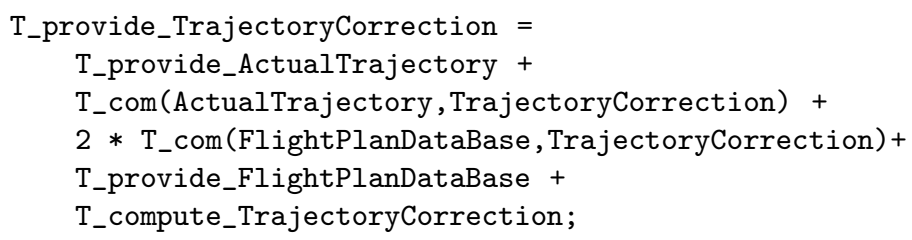

Between the ActualTrajectory and TrajectoryCorrection contexts, the communication mode is of type publish/subscribe and thus can be decomposed into T_provide_ActualTrajectory corresponding to the chain segment for computing the trajectory and T_com (ActualTrajectory, TrajectoryCorrection) corresponding to the communication time between these two contexts. Because the plannedTrajectory source of the FlightPlanDataBase is accessed by pulling the value in a synchronous manner, it is decomposed into the time to compute the data (T_provide_FlightPlanDataBase) and the communication round-trip $\left(2 * T_{-}\right.$com(FlightPlanDataBase, TrajectoryCorrection)) between the two components, assuming the size of the request and the response fit within a MTU (Maximum Transmission Unit).

Freshness and Bounded Synchronization Time. The QoS contract associated to ActualTrajectory specifies freshness and bounded synchronization time 
between InertialReferenceUnit and AirDataUnit. In the worst case, the synchronization takes the sum of the bounded synchronization time and the maximum time for receiving an event from InertialReferenceUnit or AirDataUnit. This leads to the generation of the following constraints:

T_provide_ActualTrajectory $<=2$;

T_synchronization $<=1$;

T_provide_ActualTrajectory $=$

$\max ($

T_provide_airData $+T_{-}$com(AirDataUnit, ActualTrajectory),

T_provide_localization + T_com(InertialReferenceUnit, ActualTrajectory)) +

T_synchronization +

T_compute_ActualTrajectory;

The refinement of the numerical constraints and the checking of coherence at each step ensures the coherence between all non-functional requirements. Each numerical constraint is defined using Prolog IV [9], a constraint logic programming language over real numbers, coupled with a real interval arithmetic solver for checking the coherence of each refinement step.

\subsection{From Architecture Design to Implementation}

Monitoring support is generated from the non-functional specifications. Each non-functional container is in charge of monitoring the time-related constraints associated to a given functional component. Since QoS declarations at the design level are used to generate the monitoring support, the traceability is automatically ensured between the design and implementation stages. Moreover, as this support is embedded into the programming framework, it is completely transparent to the developer. Doing so prevents the developer from introducing errors in the monitoring code. Specifically the DiaSuite exception mechanism allows to separate the detection mechanism that is generated from the treatment code that is implemented by the developer.

\subsection{From Implementation to Deployment}

During the deployment stage, the prediction tool is based on the numerical constraints generated from the QoS-extended DiaSpec specification, ensuring the traceability of the requirements. In the avionics domain, the execution platforms offer deterministic QoS characteristics (e.g., the deterministic timing of the AFDX network). Such information allows to refine the time-related constraints generated from a QoS declaration and to compare the performance of several deployment configurations.

In the generated constraints, there are several numerical variables that depend on the deployment. The $T_{-}$com_<component_name> variables depend on the communication mode. If the application is deployed on a non-distributed platform, the communication time can be considered as null between applicative components, simplifying the numerical constraints. If the application executes 
on a distributed platform, the communication time between the applicative components depends on the distributed systems technologies. In avionics, the most commonly used network is the AFDX. The communication constraints can be refined according to the AFDX bandwidth and the associated Bandwidth Allocation Gap (BAG). Concerning the communication between applicative components and devices, different sort of communication technologies can be used, such as a serial link (e.g., ARINC429, RS422) that leads to different communication times. The $\mathrm{T}_{-}$compute_<component_name> variables depend on the complexity of the algorithm and the execution platform (e.g., CPU frequency and memory access time). Finally, the T_provide_<data_sensed $>$ variables depend on sensor technologies (e.g., mechanical or LASER probes for air data).

For example, assume we enrich the system with a new functionality for displaying the trajectory on the navigation display. We want to reuse the chain segment computing the actual trajectory but with stronger QoS requirements, leading to the constraint T_provide_ActualTrajectory $<=0$.8. From all these constraints, the prediction tool infers the following value range for the Air Data

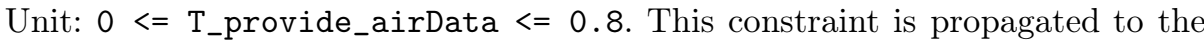
stakeholders in charge of selecting the technologies for the execution platform. In this situation, they will choose LASER probes whose performance is conform with this constraint.

\section{Towards Certification of Avionics Systems}

Our approach has been applied to the design of several avionics applications, including the flight management system, the aircraft guidance system, and the traffic collision avoidance system. These experiments have shown that the DiaSuite methodology is well-suited for the development of avionics control-loop systems. In this section, we discuss how our integrated QoS approach guides the avionics certification process.

In avionics, aircrafts have to respect the Certification Specification (CS) standard to obtain the airworthiness certificate. CS proposes the classification of the failure conditions: conditions impacting the aircraft and/or its occupants. They depend on the flight phases (e.g., landing) and the environmental conditions. Failure conditions are associated with a Design Assurance Level, indicating their degree of criticality and safety objectives. To reach safety objectives, aeronautical standards specify constraints on the development process (e.g., distribute the development stages across several teams) and the testing process (e.g., structural and black-boxes tests). These constraints cover all the levels of a system, including the software and hardware layers. Both functional and non-functional requirements 22] have to be guaranteed, including performance concerns.

Modern avionics platforms such as the Integrated Modular Avionics (IMA) allow to host several functions on the same platform [25]. The IMA approach introduces different stakeholders in the development process. The system integrator is the leading authority (generally, the airframer). The role of this stakeholder is to integrate all IMA systems: the software applications, provided by the 
function suppliers, and the hardware systems, provided by the platform suppliers. The integrator and suppliers play different roles in the certification process [12]. The system integrator specifies general constraints about all the systems at the aircraft level. For example, this stakeholder defines the Worst Case Communication Time (WCCT) of the network, ensured by the AFDX technology [4, and the WCET of the applications. These WCET requirements are passed on to the function suppliers. To fulfill them, in turn, function suppliers produce specific requirements for platform suppliers regarding issues such as time slots, CPU power, memory capacity and throughput. To do so, the platform suppliers have to provide the function suppliers with all the characteristics of this platform, including WCET for core software services (e.g., drivers and health monitoring).

From the high-level WCET constraints delivered by the system integrator, our approach guides the function suppliers in systematically specifying and refining all the non-functional requirements during the development of the application. Function suppliers can also use the generated monitoring support for validating the performance of their application. Furthermore, the prediction tool can be helpful in determining an interval of timing on each deployment technologies and passing these constraints on to the platform suppliers. The platform suppliers are now responsible for giving applications the means to access input data from the sources, and send output data to the actuators. Regarding constraints issued by all the function suppliers, the platform supplier can use the prediction tool for proposing a platform configuration that matches all their needs. Finally, the system integrator can use the generated monitoring support for both controlling the applications in flight, and performing aircraft maintenance. In doing so, information about the non-functional behavior of the application can be logged in flight and the pilot can be alerted in case of unexpected behaviors. The monitoring support can also be used on-ground for correcting errors. As this support is entirely generated, it is sufficient to test and certify the generator for guaranteeing the correctness of all future generated monitoring containers.

Requirements traceability is key to obtain avionics certification. Because the role separation proposed by the IMA platforms leads to the collaboration of several companies, requirements traceability has become significantly more challenging. The tool-based approach proposed in this paper can facilitate this certification process by offering support for propagating automatically QoS requirements between the stakeholders.

\section{Related Work}

Among existing QoS specifications languages, some of them focus on performance properties and already offer design and programming support. For example, in the spirit of our approach, Defour et al. generate numerical constraints from time-based requirements specified with the QoSCL language [10]. These constraints are not only used to check the requirements compatibility according to the architecture, but also to predict the QoS from the number of instances of each component. AlTurki et al. propose a real-time rewriting model backing a timing 
specification language [2]. It allows them to verify various real-time properties using the Maude rewriting engine. Krogmann et al. have set up a quantitative performance prediction tool into the Palladio Component Model [20, allowing architects to choose between different architectural designs. They define many types of performance requirements that would be interesting for us to take into account for critical systems (e.g., CPU). Bertolino et al. 6] also propose another performance-based prediction approach that focuses on the assembling of existing components. In contrast to our work, the above approaches are generalpurpose and are limited to the design phases of the development process.

Other approaches are domain-specific, for example dedicated to the specification of real-time systems. As a result, they can offer better design and programming support. For example, Fredriksson et al. propose a framework for leveraging non-functional requirements (e.g., time and memory consumption) to build control systems components [15] and thus to predict the functional/non-functional behavior of the composed system. Doose et al. formalize real-time systems as a set of functionalities linked within timed communications and then verify the time-coherency of the whole system using model-checking techniques [13]. Carcenac et al. also validate real-time systems according to the incremental specification of non-functional requirements [7]. Yet, these approaches only focus on the validation of the systems.

In contrast, the approach of Robert et al. enables generating monitoring support from non-functional requirements, represented as exceptional transitions in timed-automata [24. In the same spirit, Duclos et al. [14] have proposed to specify QoS requirements as aspects in the architectural models for providing separation of concerns, monitoring these requirements at runtime. The approach of Genssler et al. enables generating scheduling support based on QoS declarations 16 .

The above QoS approaches are dedicated to real-time systems and offer support at design time (e.g., prediction) and/or at runtime (e.g., monitoring). However, they are mostly dedicated to specific development stages and do not consider the traceability of non-functional concerns through the software development process.

To conclude, specifying non-functional requirements only at the architecture level is not sufficient. As observed by Koziolek et al. [19, it is crucial to clearly identify the stakeholders and the workflow between the functional development and the non-functional layer. Towards this end, we propose a unified approach that integrates QoS into the complete development process.

\section{Conclusion}

In this paper we have presented a step-wise approach integrating QoS concerns through all phases of software development. This approach dedicated to controlloop systems extends the DiaSuite tool-based methodology by offering support for specifying, validating and monitoring time-related requirements. We have shown that this domain-specific approach allows to guide the stakeholders in 
systematically refining non-functional requirements and ensures requirements traceability by generating numerical constraints. We have illustrated our approach in the avionics domain where such QoS requirements are critical.

We are currently working on a deeper evaluation of this approach with the development of an autopilot application coupled to the FlightGear simulator [23]. This work will allow to leverage DiaSpec's architectural support of error handling [21] for treating the violation of a QoS contract at runtime. In particular, we plan to show how the error handling support provided by DiaSpec can be used to implement logging and reconfiguration treatments. This evaluation would also help in refining the specification language (e.g., time constraints depending on input parameters). Future work concerns the integration of this methodology into the avionics certification process. In particular, we will need to certify our tools and their associated development approach.

\section{References}

1. Aagedal, J.Ø.: Quality of service support in development of distributed systems. PhD thesis, University of Oslo (2001)

2. AlTurki, M., Dhurjati, D., Yu, D., Chander, A., Inamura, H.: Formal specification and analysis of timing properties in software systems. In: Chechik, M., Wirsing, M. (eds.) FASE 2009. LNCS, vol. 5503, pp. 262-277. Springer, Heidelberg (2009)

3. ARINC 653, system partitioning and scheduling. Aeronautical Radio, Inc. (2003)

4. ARINC 664, AFDX: Avionics Full DupleX switched ethernet. Aeronautical Radio, Inc. (2005)

5. ARP4754, certification considerations for highly-integrated or complex aircraft systems, SAE (1996)

6. Bertolino, A., Mirandola, R.: CB-SPE tool: putting component-based performance engineering into practice. In: Crnković, I., Stafford, J.A., Schmidt, H.W., Wallnau, K. (eds.) CBSE 2004. LNCS, vol. 3054, pp. 233-248. Springer, Heidelberg (2004)

7. Carcenac, F., Boniol, F.: A formal framework for verifying distributed embedded systems based on abstraction methods. International Journal on Software Tools for Technology Transfer 8(6), 471-484 (2006)

8. Cassou, D., Bertran, B., Loriant, N., Consel, C.: A generative programming approach to developing pervasive computing systems. In: Proceedings of the 8th International Conference on Generative Programming and Component Engineering, pp. 137-146. ACM, New York (2009)

9. Colmerauer, A.: Specifications of Prolog IV (1996)

10. Defour, O., Jézéquel, J.-M., Plouzeau, N.: Extra-functional contract support in components. In: Crnković, I., Stafford, J.A., Schmidt, H.W., Wallnau, K. (eds.) CBSE 2004. LNCS, vol. 3054, pp. 217-232. Springer, Heidelberg (2004)

11. DO-178B, software considerations in airborne systems and equipment certification, RTCA, Inc. (1992)

12. DO-297, Integrated Modular Avionics (IMA) development guidance and certification considerations, RTCA, Inc. (2005)

13. Doose, D., Mammeri, Z.: Polyhedra-based approach for incremental validation of real-time systems. In: Yang, L.T., Amamiya, M., Liu, Z., Guo, M., Rammig, F.J. (eds.) EUC 2005. LNCS, vol. 3824, pp. 184-193. Springer, Heidelberg (2005) 
14. Duclos, F., Estublier, J., Morat, P.: Describing and using non-functional aspects in component-based applications. In: Proceedings of the 1st International Conference on Aspect-Oriented Software Development, pp. 65-75. ACM, New York (2002)

15. Fredriksson, J., Tivoli, M., Crnkovic, I.: A component-based development framework for supporting functional and non-functional analysis in control system design. In: Proceedings of the 20th IEEE/ACM International Conference on Automated Software Engineering, pp. 368-371. ACM, New York (2005)

16. Genssler, T., Christoph, A., Winter, M., Nierstrasz, O., Ducasse, S., Wuyts, R., Arévalo, G., Schönhage, B., Müller, P.O., Stich, C.: Components for embedded software: the PECOS approach. In: Proceedings of the Conference on Compilers, Architectures and Synthesis for Embedded Systems, pp. 19-26. ACM, New York (2002)

17. Halima, R.B., Drira, K., Jmaiel, M.: A QoS-oriented reconfigurable middleware for self-healing web services. In: Proceedings of the 6th IEEE International Conference on Web Services, pp. 104-111. IEEE, Los Alamitos (2008)

18. Jha, S., Seneviratne, A.: Synchronization skew: a QoS measurement study. In: Proceedings of the Conference on Local Computer Networks, pp. 77-78 (1999)

19. Koziolek, H., Happe, J.: A QoS driven development process model for componentbased software systems. In: Gorton, I., Heineman, G.T., Crnković, I., Schmidt, H.W., Stafford, J.A., Ren, X.-M., Wallnau, K. (eds.) CBSE 2006. LNCS, vol. 4063, pp. 336-343. Springer, Heidelberg (2006)

20. Krogmann, K., Schweda, C.M., Buckl, S., Kuperberg, M., Martens, A., Matthes, F.: Improved feedback for architectural performance prediction using software cartography visualizations. In: Mirandola, R., Gorton, I., Hofmeister, C. (eds.) QoSA 2009. LNCS, vol. 5581, pp. 52-69. Springer, Heidelberg (2009)

21. Mercadal, J., Enard, Q., Consel, C., Loriant, N.: A domain-specific approach to architecturing error handling in pervasive computing. In: Proceedings of the 25th International Conference on Systems, Programming, Languages, and Applications: Software for Humanity. ACM, New York (2010)

22. Paulitsch, M., Ruess, H., Sorea, M.: Non-functional avionics requirements. In: Proceedings of the 3rd International Symposium On Leveraging Applications of Formal Methods, Verification and Validation, pp. 369-384. Springer, Heidelberg (2009)

23. Perry, A.R.: The FlightGear flight simulator. In: Proceedings of the USENIX Annual Technical Conference (2004)

24. Robert, T., Fabre, J.-C., Roy, M.: On-line monitoring of real time applications for early error detection. In: Proceedings of the 14th IEEE Pacific Rim International Symposium on Dependable Computing, pp. 24-31. IEEE, Los Alamitos (2008)

25. Watkins, C.B., Walter, R.: Transitioning from federated avionics architectures to Integrated Modular Avionics. In: Proceedings of the 26th IEEE/AIAA Digital Avionics Systems Conference, p. 2. IEEE, Los Alamitos (2007)

26. Windsor, J., Hjortnaes, K.: Time and space partitioning in spacecraft avionics. In: Proceedings of the 3rd IEEE International Conference on Space Mission Challenges for Information Technology, pp. 13-20. IEEE, Los Alamitos (2009) 